\title{
Theology in 3D
}

Virtual Tours for Outreach and Instruction at

Pitts Theology Library

Anne Marie McLean, Emory University

Spencer Roberts, Emory University

ABSTRACT Since their introduction in the mid-1990s, virtual web tours have existed alongside traditional instruction and outreach methods as digital tools to increase access and knowledge in a variety of institutions (Mosley and Xiao 1996). By 2002, tours available on the web constituted the third most popular tour option in a survey of 111 academic libraries (Oling and Mach 2002). In the spring of 2019, Pitts Theology Library (Emory University) launched its own virtual tour project using Matterport technology to supplement instruction and outreach for students, faculty, staff, and community members. Systems \& Digital Scholarship Librarian Spencer Roberts captured and processed the 3D images to make available for the public, while Reference Librarian \& Outreach Coordinator Anne Marie McLean capitalized on the technology in volunteer projects, orientation sessions, and exhibition promotion. This session details the envisioning and execution of virtual tours of Pitts Theology Library, as well as practical applications for its use in both outreach and instruction in a theological library setting.

\section{CREATING A VIRTUAL TOUR}

In June 2019, Pitts Theology Library staff began exploring the options for creating 360-degree scans and virtual tours of the library's exhibition gallery and other spaces. Spencer Roberts, systems and digital scholarship librarian, had experience with different scanning technologies in previous positions and identified the Matterport platform as the best solution. Designed for real estate applications, Matterport comprises two components: a scanning camera with controlling iPad and an account for processing and hosting the final tours. The platform allows users to explore a virtual space using the mouse or keyboard controls to move and look around. They can also interact with tags that can include text, images, or video. Matterport also 
creates a 3D model of the space called the "Dollhouse" view and floor plans for the scanned spaces. Matterport is proprietary technology but offers a straightforward solution for virtual tours without incurring excessive costs or complicated technological skill often involved in open source approaches to 360 or 3D scanning.

Operating the Matterport is simple due to the fully contained scanning unit and intuitive control app on an iPad. The scanning unit uses a combination of high-definition and infrared cameras to capture point data and HD images. The unit is set on a tripod, placed in the space, and activated with the iPad app. The camera captures six panoramic images with 134-megapixel resolution and over 14 million points per image, rotating under its own power between each capture. During the capture, the operator can either move to stay behind the camera or else step around a corner

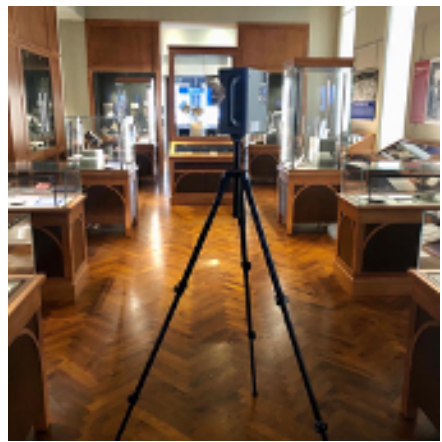

FIGURE 1: The Matterport capture unit in Pitts' exhibition gallery (photograph by Anne Marie McLean, February 3, 2020). to stay out of the images. Software then maps the scan data to stitch the images together into a seamless 360-degree view. Once the view is compiled, the operator moves the unit to a second position, usually between six and eight feet from the first position. The process is then repeated to create a second view. Ideally, spaces should be free of people and relatively static during the entire process. Objects that move or doors that change from open to closed can affect the scanning process and later navigation through the virtual tour.

The most remarkable part of the Matterport platform is how it processes and compares the point and image data from each view in order to determine their relation to one another within space. By comparing the data, the software identifies shared points and triangulates the positions of the views. Each subsequent scan added to the project is compared with previous views, allowing the whole space to be captured and mapped. The processing algorithm requires incremental progress through a space, so each scan must be close to a previous scan. This means that the user must plan out the path through and coverage of the space. However, once planned out, the scanning process is quick. The unit captures a scan in 15-20 seconds and processes in 10-15 seconds, so each view takes about 30-40 
seconds total. One of our staff members was able to scan our exhibition gallery, which is 1,200 square feet, in 30 minutes. The scanning process improves over time as the user becomes familiar with distances and positioning of the scans. Once uploaded to the Matterport servers, the scans are processed into their final form. The system automatically produces a virtual tour, a floorplan, and a "dollhouse" view. The tour, highlight reel, and MatterTags for creating interactive points of information can all be customized through the administrative web interface. For a small fee, an architectural floor plan and 3D model in OBJ format can be downloaded from the Matterport site.

FIGURE 2: The "Dollhouse" view in the Matterport user interface (screenshot by Spencer Roberts, July 15, 2020).

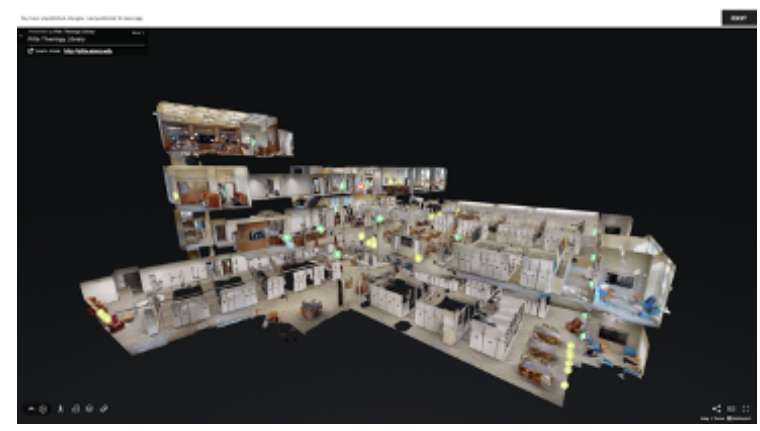

Although Matterport includes an initial investment in hardware and ongoing costs for hosting, Pitts staff were able to test the platform before investing. Emory Center for Digital Scholarship was already equipped with Matterport for their own projects, so Pitts was able to borrow their equipment to test ease of use and viability for the theology library. After a successful test, Pitts purchased its own equipment and subscription from Matterport. The software now works with a number of camera units ranging in price from $\$ 400$ to $\$ 18,000$. A recently launched beta also works with iPhone cameras, rendering 2D images into a 3D space. Pitts purchased a Matterport Pro 2 unit, which costs between $\$ 3,000$ and $\$ 4,000$, depending on discounts. The hosting plans range from $\$ 10$ to $\$ 70$ per month. Most smaller libraries would probably be comfortable with the Starter level, which allows 5 active spaces.

Pitts currently has a Professional account (up to 25 spaces) because we are scanning each of our exhibitions and also undertaking a project to capture historic rural churches in Georgia. In this project, Pitts is maintaining the scans and absorbing the cost as part of our mission. In a different partnership with the Schwartz Center 
for Performing Arts to provide views from different seats within the venue, we provided the initial scanning, hosting of the project, and continuing support for an annual fee. This model is more effective for the arts organization than attempting to implement a solution on their own and allows Pitts to offset its costs. Because Matterport collects usage statistics but not user data, Pitts can also provide the Schwartz with statistics for them to evaluate impact without compromising patron privacy.

Another benefit of the Matterport platform is the ability to transfer each project between accounts. An institution such as Pitts can use its camera to produce a scan of a smaller library or a church and then transfer that project to an account held by a different organization that does not own its own hardware. This allows partners with smaller budgets to subscribe to a lower account level and not incur the costs for equipment. Larger institutions such as Pitts can serve as facilitators to allow smaller organizations to produce similar tours for their own outreach and instruction.

There are two technological drawbacks to the Matterport system that Pitts identified. The first issue is that the core file formats for the Matterport tours are proprietary and cannot be ported to other systems or preserved. We consider this a reasonable limitation when compared to the ease and quality of the system. The second issue is the lack of compatibility with screen readers and other accessibility tools. Although some of the text content in the virtual tour can be read with a screen reader, navigation through the space is entirely visual. This limitation is common in many virtual space applications or interfaces, but we plan to explore ways of providing similar experiences that are accessible for sense-impaired users.

\section{VIRTUAL TOURS IN OUTREACH AND INSTRUCTION}

Pitts Theology Library has a rich history of strategic outreach through exhibitions, events, and instruction, bringing together Emory faculty, students, staff, community members, and outside organizations (Bedard 2016). However, these methods of outreach were reliant on participatory frameworks that required users to enter the building and engage with the space. Given the rising trend of virtual alternatives, in addition to the drastic shift to remote learning and research as a result of the Covid-19 pandemic, Pitts' engagement with more 
accessible alternatives through virtual tours was long overdue (Oling and Mach 2002).

Virtual tours are first and foremost crucial from an accessibility perspective. The Emory Library Accessibility Committee documents and continues to address the challenges of visitor and handicap access to the campus buildings situated in the congested city of Atlanta, including parking, elevator options, and turnstiles. Pitts Theology Library's virtual tour option bypasses these challenges by bringing the space to the user, as opposed to requiring the user to plan what often results in a day-long excursion to the space. This alternative lends itself to those confined to learning from any distance, figuratively or literally. In addition, the virtual tour alternative can be cost effective for visitors who would otherwise be required to pay for visitor parking, meals, and transportation. Candidates for these pecuniary benefits might include school groups with limited funding for field trips, researchers on a meager stipend, or even life-long learners with a narrow income.

While the accessibility generated by the virtual tours was primarily conceived with pedagogical ends in mind, it also lends itself to unanticipated user groups. Because Pitts Theology Library was recently redesigned as a partition of the larger Candler School of Theology in 2014, it has been a point of interest for other libraries and exhibition galleries in the process of redesign. Since the building's opening, architecture and interior design firms continue to make on-site visit requests. The "dollhouse," "floorplan," and "measurement mode" options in the Matterport interface are particularly attractive for this audience. In addition, after new state tax incentives were offered in 2008 to stimulate the film industry in Georgia, the industry's presence on Emory's campus increased dramatically (see Emory University n.d.). Pitts Theology Library is no exception and was considered as a location for the Marvel film "Ant-Man and the Wasp" in the summer of 2018 (Weiner 2018). With the new virtual tour option in place, Pitts hopes to engender more interest from professional firms, location scouts, and more as a convenient alternative to scheduling in-person visits.

In addition to rendering the physical space of the library accessible, the virtual tours also allow for a detailed glimpse into services that the library provides, as well as an in-depth look at objects in Pitts. Points of interest are assigned spatial tags that allow the user to learn more about the services provided at that location, whether it be the 
reference desk or group study rooms. This feature can supplement or even replace traditional "scavenger hunts" and tours given for new student orientation, allowing users to glean the same information without traversing the building (Scott and Gregor 2013). Even objects throughout the library become interactive through the virtual tour. Friend of the library Ginny Connelly noted that, when she started volunteering at Pitts,

The beautiful building was new and the library and collections were new to me as well. Much of the art and other displays were unlabeled. I wanted to compile information about the graphics, photos, prints, paintings, scrolls-all the visual enhancements throughout the building-and somehow make this available to library volunteers and visitors to enrich their experience of the library. (Ginny Connelly, pers. comm., June 3, 2020)

The Matterport technology and spatial tagging gave Ginny and other volunteers an outlet for communicating this information, and they continue to gather metadata on artwork around the building. Along those same lines, spatial tagging has become a key component in the development of digital exhibitions at Pitts Theology Library. While cases and objects currently repeat the descriptions printed on the physical gatorboard labels, options to zoom in on objects, play videos, and link out to external resources, such as flipbook views or interactive maps, are promising features for future exhibitions.

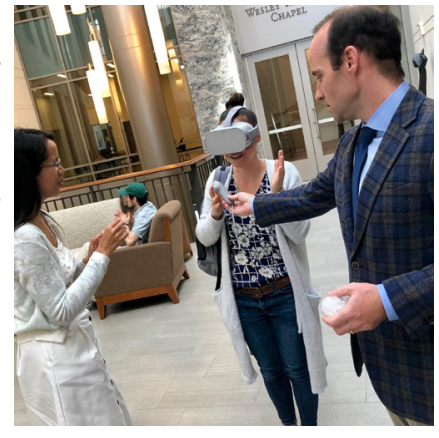

FIGURE 3: Emory graduate students take a virtual tour of the new exhibition via an Oculus Go VR headset (photograph by Anne Marie McLean, September $26,2019)$.

While not by design, the introduction of virtual tours at Pitts Theology Library coincided with a critical point in secondary education as institutions shifted to remote learning and research. Armed with new tools and an even broader notion of what it means to make the library truly accessible, Pitts looks forward to harnessing Matterport technology and virtual tours as part of its mission to preserve, interpret, and provide access to information resources for not only the Candler School of Theology but learners across the globe. 


\section{REFERENCES}

Ashmore, Beth, and Jill E. Grogg. 2004. "Library Virtual Tours: A Case Study.” Research Strategies 20, no. 1: 77-88. doi.org/10.1016/j. resstr.2005.10.003.

Bedard, Rebekah. 2016. "Tapping Outside Interest in Special Collections: Collaborative Outreach Initiatives at Pitts Theology Library.” Atla Summary of Proceedings 70: 257-67.

Duncan, James M. and Linda K. Roth. 2001. "Production of the NextGeneration Library Virtual Tour.” Bulletin of the Medical Library Association 89, no. 4 (October): 331-8.

Emory Center for Digital Scholarship. n.d. “About Us.” Accessed July 15, 2020. digitalscholarship.emory.edu/about/index.html.

Emory University. n.d. "Emory in Film \& TV.” Accessed June 15, 2020. filming.emory.edu/EmoryinFilm/index.html.

Hickok, John. 2002. "Web Library Tours: Using Streaming Video and Interactive Quizzes.” Reference Services Review, June 1, 2002. doi. org/10.1108/00907320210428660.

Mornini, Joelle. 2018. "8 Examples of Virtual Library Tours." The Always Learning Librarian, March 18, 2018. alwayslearninglibrarian.wordpress.com/2018/03/18/8-examples-of-virtual-librarytours.

Rice, Scott, and Margaret N. Gregor. 2013. "This Library Orientation Is Fun! : Building a Successful Virtual Tour Experience for Students.” ACRL 2013, 6. www.ala.org/acrl/sites/ala.org.acrl/files/ content/conferences/confsandpreconfs/2013/papers/RiceGregor ThisLibrary.pdf.

Mosley, Pixey Anne and Daniel Xiao. 1996. "Touring the Campus Library from the World Wide Web.” Reference Services Review 24: 7-14.

Oling, Lori and Michelle Mach. 2002. "Tour Trends in Academic ARL Libraries.” College \& Research Libraries 63, no. 1: 13-23.

Schwartz Center for Performing Arts. n.d. "View from Your Seat." Accessed July 15, 2020. schwartz.emory.edu/events-tickets/boxoffice-information/view-from-your-seat.html. 
Weiner, Jesse. 2018. “'Ant-Man and the Wasp' A Small But Mighty Sequel.” The Emory Wheel, July 11, 2018. emorywheel.com/antman-and-the-wasp-a-small-but-mighty-sequel. 